\title{
The Japanese reporting system for thyroid aspiration cytology 2019 (JRSTAC2019)
}

\author{
Mitsuyoshi Hirokawa ${ }^{1}$, Ayana Suzuki ${ }^{1}$, Miyoko Higuchi ${ }^{1}$, Toshitetsu Hayashi ${ }^{1}$, Seiji Kuma ${ }^{1}$, Yasuhiro Ito ${ }^{2}$, \\ Akira Miyauchi
}

${ }^{1}$ Department of Diagnostic Pathology and Cytology, Kuma Hospital, Kobe, Japan; ${ }^{2}$ Department of Surgery, Kuma Hospital, Kobe, Japan Contributions: (I) Conception and design: M Hirokawa; (II) Administrative support: M Hirokawa, T Hayashi, S Kuma; (III) Provision of study materials or patients: M Hirokawa, A Suzuki, M Higuchi; (IV) Collection and assembly of data: M Hirokawa, A Miyauchi, Y Ito; (V) Data analysis and interpretation: M Hirokawa, A Suzuki, Y Ito, A Miyauchi; (VI) Manuscript writing: All authors; (VII) Final approval of manuscript: All authors. Correspondence to: Mitsuyoshi Hirokawa, MD, PhD, FIAC. Department of Diagnostic Pathology and Cytology, Kuma Hospital, 8-2-35 ShimoyamateDori, Chuo-Ku, Kobe, Hyogo 650-0011, Japan. Email: mhirokawa@kuma-h.or.jp.

\begin{abstract}
We introduce the Japanese reporting system for thyroid aspiration cytology 2019 (JRSTAC2019) proposed by the Japan Association of Endocrine Surgery and the Japanese Society of Thyroid Pathology. Pathological classification and recommended clinical management for thyroid nodules in Japan are different from those described in the World Health Organization classification or the Bethesda System for Reporting Thyroid Cytopathology (TBSRTC). Therefore, it was necessary to develop a reporting system adapted for Japan. JRSTAC2019 is a modified version of TBSRTC. Currently, JRSTAC2019 is widely used in Japan, although the details of the system have not been introduced in English. JRSTAC2019 comprises seven categories: (I) unsatisfactory, (II) cyst fluid, (III) benign, (IV) undetermined significance, (V) follicular neoplasm (FN), (VI) suspicious for malignancy (SFM), and (VII) malignant. "Cyst fluid" nodules are classified as an independent category, and "recommended management" is in the same category as "benign" nodules. Surgical resection for FN nodules is decided upon by considering several parameters, and the decision is made without performing gene analysis. Non-invasive follicular thyroid neoplasm with papillarylike nuclear features tends to be more often diagnosed as papillary carcinoma. The risk of malignancy of SFM in Japan is higher than that in Western countries, and resection rates of SFM and malignant tumors are lower owing to active surveillance for low-risk papillary microcarcinoma. We recommend that each country should develop its reporting system, suitable for its medical and social needs. However, it should be easily compatible with TBSRTC for the ease of academic data sharing.
\end{abstract}

Keywords: Bethesda system; cyst fluid; fine-needle aspiration cytology; reporting system; thyroid

Submitted Apr 06, 2020. Accepted for publication Sep 02, 2020.

doi: $10.21037 /$ gs-2019-catp-22

View this article at: http://dx.doi.org/10.21037/gs-2019-catp-22

\section{Introduction}

It is essential for institutions performing thyroid fineneedle aspiration (FNA) to share the terminology and diagnostic criteria for reporting. In 2007, the Bethesda System for Reporting Thyroid Cytopathology (TBSRTC) was proposed as a reporting system for thyroid FNA specimens (1). The 2015 American Thyroid Association (ATA) management guidelines endorsed the system for adult patients with thyroid nodules and differentiated thyroid cancer (2) and have been widely adopted globally. However, some countries have not accepted the system in its entirety. England, Italy, and Australia have used their reporting systems (3-5).

Similarly, Japanese reporting systems have also been proposed as the pathological classification and clinical management of thyroid tumors are different from the practices in the West. Here, we introduce the Japanese 
reporting system for thyroid aspiration cytology 2019 (JRSTAC2019) proposed by the Japan Association of Endocrine Surgery (JAES) and the Japanese Society of Thyroid Pathology (JSTP) (6). Currently, JRSTAC2019 is the universally used reporting system in Japan, but its details have not been introduced in English.

\section{Development of the reporting system for thyroid FNA in Japan}

Table 1 shows reporting systems for thyroid FNA used in Japan. A reporting system for thyroid FNA was first proposed by the Papanicolaou Society of Cytopathology Task Force on Standards of Practice in 1996 (7). It consists of seven categories: (I) unsatisfactory for interpretation, (II) benign non-neoplastic lesions, (III) cellular follicular lesions, (IV) FN, (V) Hürthle cell neoplasm, (VI) SFM, and (VII) malignant. In 2005, the Japanese Society of Thyroid Surgery (JSTS) modified the proposal and published JRSTAC2005 that comprised five categories: (I) inadequate, (II) normal or benign, (III) intermediate, (IV) malignancy suspected, and (V) malignant (8). In 2007, TBSRTC established a standardized, category-based reporting system for thyroid FNA (1). Subsequently, TBSRTC was endorsed by ATA and has been widely adopted in the United States and worldwide (2). In 2013, the Japan Thyroid Association (JTA) proposed an original diagnostic system for reporting thyroid cytology (JTA2013) (9). In JTA2013, "cyst fluid" was categorized under "benign", and "follicular neoplasm (FN)" was subclassified into (I) favor benign, (II) borderline, and (III) favor malignant, which was adapted from classifications used in two high-volume thyroid disease centers. However, JTA2013 was not widely accepted because general cytopathologists with limited experience with thyroid diseases were unable to differentiate well enough to subclassify follicular neoplasms. Two years later, JSTS revised the JRSTAC2005 to produce JRSTAC2015 (10), which comprised seven categories: (I) unsatisfactory, (II) cyst fluid, (III) benign, (IV) undetermined significance, (V) FN, (VI) SFM and (VII) malignant. In 2018, the JSTS was dissolved, and the activities it performed were taken over by the JAES. In 2019, the system was again revised (JRSTAC2019) by both the JAES and JSTP (6), but the terms and criteria of the seven categories remained unchanged. Currently, although both reporting systems JTA2013 and JRSTAC2019 are available in Japan, the latter is more popular.

\section{Diagnostic categories of JRSTAC2019}

\section{Unsatisfactory}

Thyroid FNA samples should be evaluated for specimen adequacy (Table 2). If the samples do not fall into any of the following four scenarios representing adequacy, they are categorized as "unsatisfactory":

(I) A minimum of six groups of well-visualized follicular cells, with at least 10 cells per group;

(II) Abundant colloid;

(III) Cells with significant cytological atypia;

(IV) Inflammatory cells, including lymphocytes, plasma cells, or histiocytes.

Specimens with more than just histiocytes that are obtained from a solid area, as well as foamy histiocytes obtained from the cystic fluid, are regarded as adequate. Poorly preserved specimens, including degeneration due to desiccation, poor fixation, obscuring blood, clotting artifacts, and smearing failures, are also categorized as "unsatisfactory". It must be noted. However, blood components, skeletal muscle, and ciliated cells are not to be included in the diagnostic criteria. The reason/ s for categorizing specimens as "unsatisfactory", should be specified in the cytological report. A clinical recommendation for this category is a repeat aspiration, if shown. The frequency of aspirated nodules categorized as "unsatisfactory" is recommended to be $\leq 10 \%$.

\section{Cyst fluid}

The samples obtained from cystic fluid materials are included in this category. The samples often include many foamy histiocytes. Colloid materials are few or absent. If there are more than six groups with 10 benign follicular cells in the preparations obtained from cystic fluid materials, samples are categorized as benign. Preparations containing histiocytes that are considered aspirated from solid areas are not included in this category; the definition of this category is the same as the subcategory of TBSRTC, "cyst fluid only" (CFO). Most samples categorized as "cyst fluid" are benign cystic lesions. However, the possibility of cystic papillary thyroid carcinoma (PTC) cannot be completely ruled out. Sonographic follow-up is recommended. If the ultrasound examination reveals a solid area in the cystic lesions, then repeated FNA for the solid area is recommended. 


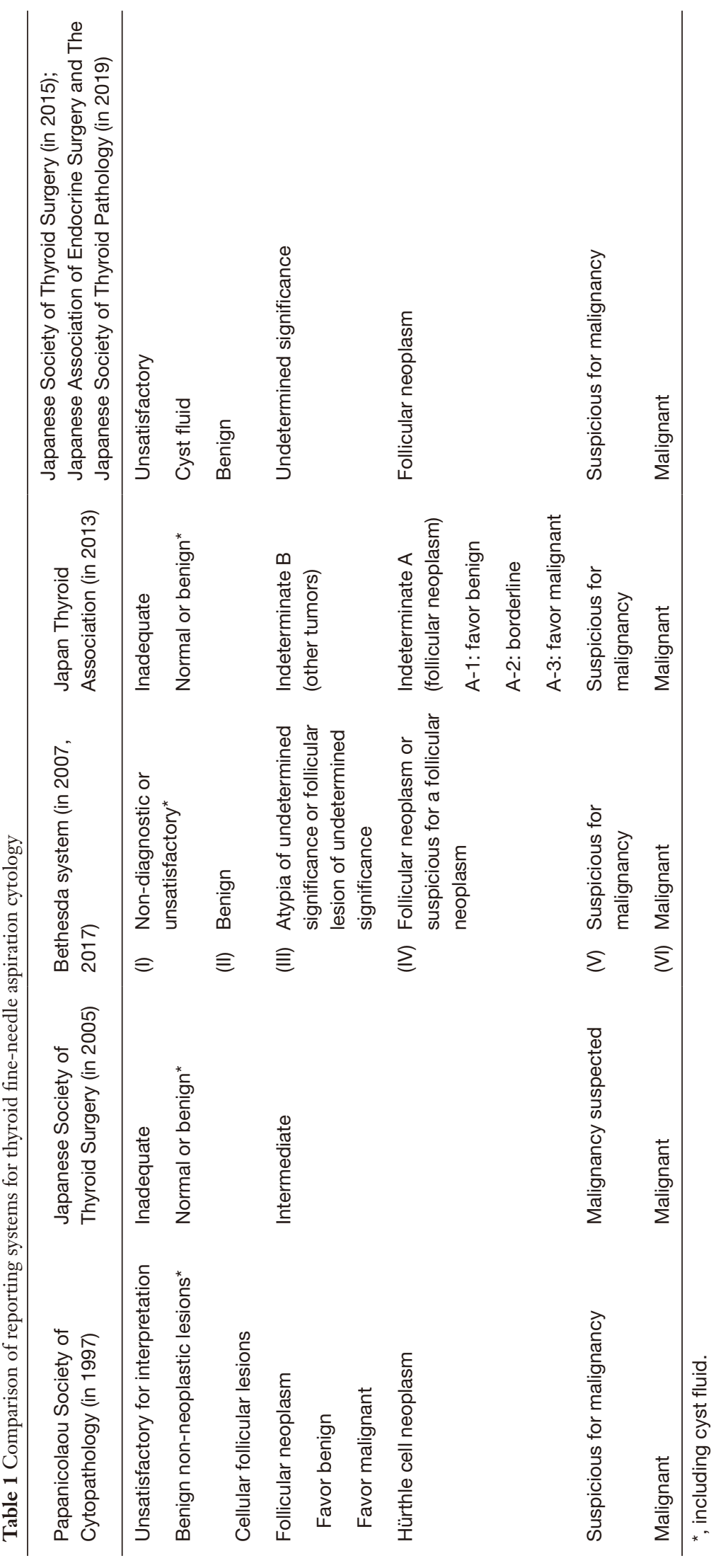


Table 2 Criteria for adequacy and unsatisfactory in the Japanese reporting system for thyroid aspiration cytology 2019

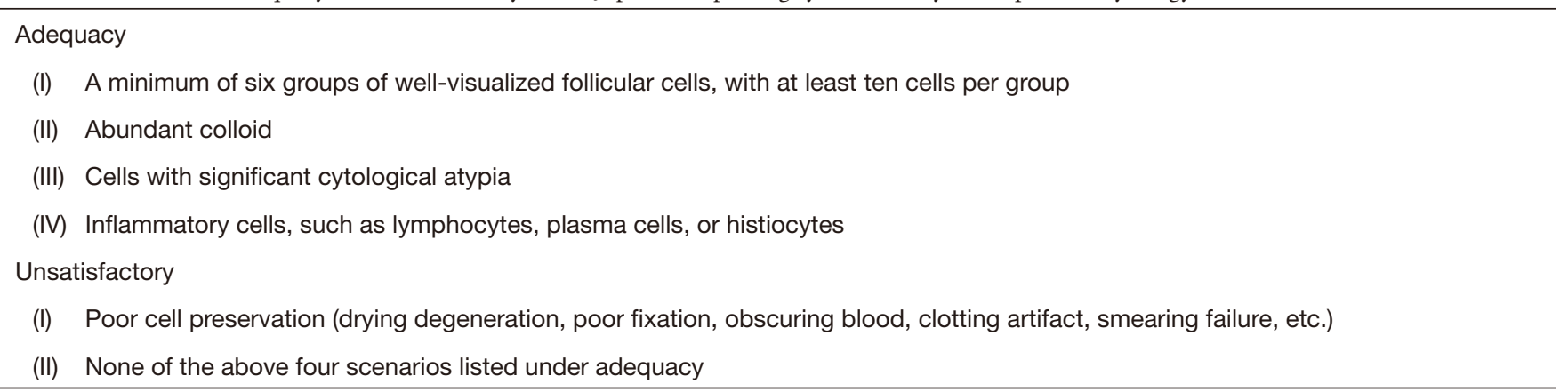

Table 3 Cases reported as undetermined significance in the Japanese reporting system for thyroid aspiration cytology 2019

(I) Papillary carcinoma is possible or not excluded

Few papillary carcinoma-suspected cells or components (pseudo inclusions, nuclear grooves, psammoma bodies, septate intracytoplasmic vacuoles, papillary structure)

Atypical cells in benign follicular tumor

Atypical cells in chronic thyroiditis

Atypical cells in cystic fluid material

Psammoma bodies in benign follicular lesions

Cellular sample with mild nuclear features of papillary carcinoma

(II) Medullary carcinoma is possible or not excluded

Salt and pepper chromatin, extremely eccentric nuclei, amyloid materials, spindle cells, large hyperchromatic nuclei

(III) Undetermined atypical cells, Undetermined mesenchymal cells

(IV) Hashimoto thyroiditis or lymphoma

(V) Benign lesion or malignant lesion, Unknown tumorous lesion

\section{Benign}

Adequate samples with no malignant cells are categorized as "benign". The samples have benign follicular cells, considerable numbers of inflammatory cells, or abundant colloid materials. The benign follicular cells are arranged in monolayered sheets, which have a honeycomb pattern but can also show three-dimensional variably sized follicular patterns. A cohesive papillary pattern not associated with the nuclear features of PTC can be observed. Materials having clusters of histiocytes without cystic fluid components are included in this category. This category includes normal thyroid, nodular goiter, colloid nodule, hyperplastic nodule, acute thyroiditis, subacute thyroiditis, chronic thyroiditis (Hashimoto thyroiditis), Riedel thyroiditis, Graves' disease, etc. IgG4 related thyroiditis, most cases of which lack systemic manifestations, is included in this category.
Clinical management includes follow-up. If the nodules reveal a suspicious ultrasonographic pattern, then repeat FNA should be considered.

\section{Undetermined significance}

This category includes nodules that are challenging distinguishing between benign and malignant, or nodules containing atypical cells where a possibility of malignancy cannot be excluded. They may be follicular cells, lymphoid cells, or other unknown atypical cells. These atypical cells are insufficient cellular and/or architectural atypia to be classified as FN, SFM, or malignant. FN-suspected nodules are not included in this category. Table 3 shows cases classified in this category; most are unexcluded PTC nodules. Typically, a few atypical cells with nuclear features of PTC are present in a background of benign thyroid 
lesions, and cellular samples with mild nuclear features of PTC are also included in this category. Microfollicular lesions with mild to moderate nuclear features of PTC can be classified as "undetermined significance", "FN", or "SFM", depending on the degree of the nuclear findings and the view of observers. The following types of nodules are also included in this category: those with undetermined atypical cells or undetermined mesenchymal cells, those that are challenging distinguishing between chronic thyroiditis and lymphoma, and those where it is impossible to exclude a possibility of medullary carcinoma. Clinical management of this category is repeat FNA. However, for nodules with the possibility of medullary carcinoma or lymphoma, a measurement of serum calcitonin and flow cytometry are recommended, respectively. The recommended frequency of nodules with "undetermined significance" is $\leq 10 \%$ of adequate samples.

\section{FN}

FN refers to the nodule in which follicular adenoma or follicular carcinoma is present or suspected. Hürthle cell (oncocytic) type adenoma and carcinoma are also included in this category. The samples are cellular and are composed of monotonous follicular cells with a microfollicular pattern, where the follicular cells do not exhibit nuclear features of PTC. The background is usually hemorrhagic, and a watery colloid is not observed. The Hürthle cell type may be associated with foamy histiocytes in the background. Most nodules classified into this category are follicular adenoma or follicular carcinoma, but the benign follicular nodule, the follicular variant of PTC, or parathyroid adenoma could also fall into this category. Follicular adenomas with bizarre nuclei are classified as FN but may also be classified as "undetermined significance" or "SFM", depending on the quantity and degree of the bizarre cells. Repeated aspiration for the nodules classified into this category is not recommended because the possibility that the cells would change to another category with repeated aspiration is quite low. The recommended frequency of nodules with $\mathrm{FN}$ is $\leq 10 \%$ of adequate samples.

\section{$S F M$}

Nodules that are quantitatively or qualitatively insufficient for a definitive malignant diagnosis are categorized as SFM. This category includes various malignant tumors, and most of them are suspicious for PTC. Hyalinizing trabecular tumor is also included in this category. Nodules suspicious of FN are not included in this category. Follicular adenoma with bizarre nuclei, nodular goiter, and chronic thyroiditis may be included. Although the recommended clinical management is surgical resection, active surveillance for nodules with suggested low-risk papillary microcarcinoma is acceptable. It is recommended that more than $80 \%$ of nodules with SFM should be proved to be malignant by histological examination.

\section{Malignant}

This category is applied when the cytological features are conclusive for malignancy. It is imperative to classify the malignancy in the report, including PTC, poorly differentiated carcinoma, medullary carcinoma, anaplastic carcinoma, metastatic carcinoma, lymphoma, etc. In PTC nodules, the description of the variant can be beneficial in its clinical management. Although the recommended clinical management is surgical resection, active surveillance for nodules with suggested low-risk papillary microcarcinoma is also acceptable.

\section{Comparison of JRSTAC2019 and TBSRTC}

\section{Terminology}

The terms of the categories used in JRSTAC2019 are different from those in TBSRTC. Similarly, the UK Royal College of Pathologists (3), the Italian Society of Anatomic Pathology and Diagnostic Cytology (4), and the Royal College of Pathologists of Australia and the Australian Society of Cytology (5) use different terms. However, the categories and abbreviations proposed by TBSRTC are widely used across the world. The abbreviations may confuse those who are not accustomed to thyroid cytology. Also, some categories have two terms for the same cytological observation, e.g., "atypia of undetermined significance (AUS)" or "follicular lesion of undetermined significance (FLUS)". TBSRTC states the term AUS is preferred, although FLUS is an acceptable alternative for the vast majority of cases in which the atypia was of follicular cell origin. To avoid confusion, it would be better if each category consisted of a single term. Due to this, the terms used in JRSTAC2019 are simple and have been widely accepted by Japanese cytopathologists and cytotechnologists.

Another difference between the systems is that there 
are six categories used in TBSRTC, while there are seven in JRSTAC2019; however, this is not a significant difference. A mass consisting of "unsatisfactory" and "cyst fluid" in JRSTAC2019 is comparable with "nondiagnostic or unsatisfactory (ND/UNS)" in TBSRTC. Therefore, because the systems are not that different in their categorization terms, it is simple to share data among institutions using both the JRSTAC2019 and TBSRTC.

\section{CFO nodules}

In the "Guidelines of the Papanicolaou Society", published in 1996, CFO was reported as "consistent with benign thyroid cyst" (7). According to TBSRTC, as the possibility of cystic PTC cannot be excluded in CFO nodules, the cases are classified as ND/UNS (1). Similarly, the Italian and British Reporting System respectively classified CFO into the non-diagnostic category as TIR $1 \mathrm{C}$ and Thy1c $(3,4)$. In the Australian system, cyst fluids are classified into the non-diagnostic or benign category on clinical findings (11). In JRSTAC2005 and JTA2013, CFO nodules are categorized under "benign" $(8,9)$. In Japan, the incidence of malignancy in CFO nodules was extremely low $(0.2 \%)(12)$, which was lower than those of ND/ UNS nodules, excluding CFO (5.6\%) and benign nodules $(1.2 \%)(13,14)$. Consequently, CFO nodules are presently listed as an independent category in JRSTAC2019 (6). The follow-up is determined by risk stratification on ultrasound patterns, the same as for "benign" nodules recommended by ATA guidelines (2).

\section{Non-invasive follicular thyroid neoplasm with papillary- like nuclear features (NIFTP)}

In revised TBSRTC, the diagnostic criteria for FN/ suspicious for a follicular neoplasm (SFN) were revised considering NIFTP (15). Follicular lesions showing mild nuclear changes associated with PTC were included in FN/SFN. Also, to avoid false positives due to NIFTP, the diagnosis of PTC was limited to using nodules with classical features of PTC (true papillae, psammoma bodies, and nuclear pseudoinclusions). In preoperative cytology, most NIFTPs are diagnosed as FN/SFN, AUS/FLUS, or SFM (15-18). According to a review by Faquin et al., the preoperative cytological diagnoses of NIFTPs were ND/ UNS (0.6\%), benign (8.7\%), AUS/FLUS (31.2\%), FN/ SFN (26.6\%), SFM (24.3\%), and malignant (8.7\%) (16). More recently, the prevalence of NIFTP among lesions with FNAC of SFM and malignant tended to be lower (14\% and $3 \%$, respectively) (19). In Japan, the histological diagnosis of thyroid tumors is on "General Rules for the Description Thyroid Cancer, 8th edition" proposed by JAES and JSTP (6). The borderline tumor entity, NIFTP, was not adopted in the new classification system. Japanese pathologists tended to diagnose NIFTP as a follicular adenoma (FA) for a non-invasive encapsulated follicular tumor with focal or mild nuclear features of PTC; however, $29.5 \%$ of the tumor diagnosed as FA was later found to be NIFTP $(20,21)$. Then, tumors diagnosed as NIFTP in Western countries are classified as either FA or noninvasive encapsulated follicular variants of PTC in Japan. Cytologically, in Japan, most of the nodules equivalent to NIFTP are classified into FN or malignant, depending on the severity of nuclear features. According to the reports from Kuma Hospital, a high-volume thyroid center, 54.5\% and $45.5 \%$ of NIFTPs diagnosed as FA were categorized into AUS/FLUS and FN/SFN, respectively, and $65.9 \%$ of NIFTPs diagnosed as PTC were categorized as malignant $(21,22)$. This shows that NIFTP in Japan tends to be more often diagnosed as PTC than that in Western countries $(16,17)$. Also, in Japan, revising TBSRTC did not cause major changes in the incidence of each diagnostic category due to the low incidence of NIFTP (23). For this, it was considered unnecessary to include this category in the new classification system.

\section{Risk of malignancy (ROM)}

In revised TBSRTC (14), implied ROMs were recalculated, on post-2010 data, in two instances: when NIFTP was considered a malignancy and when it was not. Table 4 shows the incidence, resection, and malignancy rates of each category in TBSRTC alongside those observed in Kuma Hospital with JRSTAC2019 (12-15,24-27). There was no significant difference between the corresponding categories for each system, except for ROM of SFM nodules in Kuma Hospital, as these rates were found to be high (>95\%). JRSTAC2019 recommends that more than $80 \%$ of SFM nodules should be proved to be malignant through histological examination (6). However, ROM of SFM nodules in TBSRTC is approximately $50 \%$ (15). This may be because Western cytopathologists may be more prone to over-diagnosis than those in Japan. The resulting ROMs for a patient diagnosed with AUS/FLUS and FN/SFN in TBSRTC were $6-18 \%$ and $10-40 \%$, respectively (15). The ROMs are wide. TBSRTC described 
Table 4 Incidence, resection rates, and malignancy rates of Bethesda system 2017 and Kuma Hospital according to Japanese reporting system 2019

\begin{tabular}{|c|c|c|c|}
\hline Categories & Incidence, \% & Resection rates, \% & Malignancy rates \\
\hline \multicolumn{4}{|l|}{ Bethesda system 2017} \\
\hline Nondiagnostic/unsatisfactory & $3-34$ & - & All: 5-10\%; Resected: 9-32\% \\
\hline Benign & $60-70$ & 10 & All: $0-3 \%$ \\
\hline $\begin{array}{l}\text { Atypia of undetermined significance or follicular } \\
\text { lesion of undetermined significance }\end{array}$ & $1-22$ & - & $6-18 \%$ \\
\hline Suspicious for malignancy & $1.0-6.3$ & - & $45-60 \%$ \\
\hline Malignant & $2-16$ & - & $94-96 \%$ \\
\hline \multicolumn{4}{|l|}{ Japanese reporting system 2019 (Kuma Hospital) } \\
\hline Undetermined significance & $2.2-3.9$ & $16.5-25.8$ & All: $13.1 \%$; Resected: $60.8-79.5 \%$ \\
\hline Follicular neoplasm & $3.9-4.3$ & $42.2-51.0$ & All: 11.4\%; Resected: $22.3-26.2 \%$ \\
\hline Suspicious for malignancy & $1.5-2.2$ & $57.9-64.5$ & Resected: $95.8-98.8 \%$ \\
\hline Malignant & $12.2-16.4$ & $68.3-72.1$ & Resected: 99.2-99.3\% \\
\hline
\end{tabular}

AUS/FLUS with cytologic atypia was PTC in $28-56 \%$ of cases, and $27-68 \%$ of resected FN/SFN nodules were PTC (15). In our experience, the incidence of PTCs in resected AUS/FLUS and FN/SFN nodules was $44.3 \%$ and $4.6 \%$, respectively (27). Notably, the incidence of PTC among FN/SFN nodules in Japan is considerably lower than those in Western countries. This incidence is not surprising, as ROMs and incidence in each category are affected by various factors including histological and cytological diagnostic criteria, the experience and philosophy of cytopathologists, indication of surgical excision, institutions, race, country, health care system structures, and social backgrounds. After this, ROMs were not estimated in JRSTAC2019.

\section{Clinical management}

Clinical management in Japan is different from that in Western countries. The recommended management of FN/ SFN nodules is surgical excision in Western countries, and the option of molecular testing is incorporated before going ahead directly to surgery (15). In Japan, not all FN nodules underwent surgical resection. Half of FN nodules have been followed up without molecular testing $(27,28)$. Immediate surgery for $\mathrm{FN}$ is recommended for patients with the following clinicopathological findings: cytological atypia or ultrasound findings with a possibility of malignancy, large tumor size, serum level of thyroglobulin $>1,000 \mathrm{ng} / \mathrm{mL}$, nodules strongly compressing the trachea or esophagus, nodules expanding into the mediastinum, autonomously functioning nodules or nodules with cosmetic problems $(29,30)$. At high-volume thyroid disease centers, the sub-categorization (favor benign, borderline, and favor malignant) of $\mathrm{FN}$ nodules has been performed to reduce unnecessary diagnostic thyroidectomy $(9,31,32)$. ROMs for patients diagnosed with the favor benign, borderline, and favor malignant subtypes were $5-15 \%, 5-30 \%$, and $40-60 \%$, respectively. For FN nodules with the favor of malignant subtype, surgical resection is recommended. For the benign and borderline subtypes, clinical management is determined by considering other findings. Vuong et al. proved the lower resection rates and higher ROMs for FN nodules in Asian countries compared with those in Western countries (33). Consequently, Japanese clinicians tend to adopt a more conservative approach, while immediate diagnostic surgery is favored in Western practice. Currently, there is no institution performing molecular testing for aspirated materials in Japan. Also, active surveillance 
for adult patients with low-risk papillary thyroid microcarcinoma is the accepted treatment strategy in Japan (34). Therefore, resection rates of SFM and malignant nodules were $57.9-64.5 \%$ and $68.3-72.1 \%$, respectively (Table 4). The resection rates typically depend on the proportion of low-risk papillary thyroid microcarcinoma that can undergo active surveillance. Active surveillance is still a minor choice in Western countries. Therefore, JRSTAC2019 could not adopt the clinical management recommendations of TBSRTC. Overall, it is critical to understand that clinical management strategies between Japan and Western countries differ, resulting in different statistics.

\section{Conclusions}

Pathological classification and recommended clinical management for thyroid nodules in Japan differ from those described in the WHO classification and TBSRTC. Therefore, it was necessary to develop a reporting system adapted for Japan. JRSTAC2019, extensively used in Japan, is a modified version of TBSRTC. The significant differences in this system are that it forms of seven categories with simple terms. CFO nodules are classified as an independent category, and NIFTP tends to be more often diagnosed as PTC.

Further, the surgical indication for FN nodules is decided by consideration of several parameters without gene analysis. The ROM of SFM is also considerably high, and resection rates of SFM and malignant were found to be lower due to active surveillance for low-risk papillary microcarcinoma. We recommend each country develop its reporting system, suitable for its medical and social needs. However, it should be easily compatible with TBSRTC for the ease of academic data sharing.

\section{Acknowledgments}

Funding: None.

\section{Footnote}

Provenance and Peer Review: This article was commissioned by the Guest Editor (Kennichi Kakudo) for the series "Asian and Western Practice in Thyroid Pathology: Similarities and Differences" published in Gland Surgery. The article has undergone external peer review.
Conflicts of Interest: All authors have completed the ICMJE uniform disclosure form (available at http://dx.doi. org/10.21037/gs-2019-catp-22). The series "Asian and Western Practice in Thyroid Pathology: Similarities and Differences" was commissioned by the editorial office without any funding or sponsorship. The authors have no other conflicts of interest to declare.

Ethical Statement: The authors are accountable for all aspects of the work in ensuring that questions related to the accuracy or integrity of any part of the work are appropriately investigated and resolved.

Open Access Statement: This is an Open Access article distributed in accordance with the Creative Commons Attribution-NonCommercial-NoDerivs 4.0 International License (CC BY-NC-ND 4.0), which permits the noncommercial replication and distribution of the article with the strict proviso that no changes or edits are made and the original work is properly cited (including links to both the formal publication through the relevant DOI and the license). See: https://creativecommons.org/licenses/by-nc-nd/4.0/.

\section{References}

1. Cibas ES, Ali SZ. The Bethesda system for reporting thyroid cytopathology. Thyroid 2009;19:1159-65.

2. Haugen BR, Alexander EK, Bible KC, et al. 2015 American thyroid association management guidelines for adult patients with thyroid nodules and differentiated thyroid cancer: The American thyroid association guidelines task force on thyroid nodules and differentiated thyroid cancer. Thyroid 2016;26:1-133.

3. Perros P, Boelaert K, Colley S, et al. British thyroid association. Guidelines for the management of thyroid cancer. Clin Endocrinol (Oxf) 2014;81 Suppl 1:1-122.

4. Nardi F, Basolo F, Crescenzi A, et al. Italian consensus for the classification and reporting of thyroid cytology. J Endocrinol Invest 2014;37:593-9.

5. Kumarasinghe MP, Cummings $\mathrm{MC}$, Raymond $\mathrm{W}$, et al. Approach to thyroid cytology: rationale for standardisation. Pathology 2015;47:285-8.

6. Japan association of endocrine surgery, The Japanese society of thyroid pathology. General rules for the description of thyroid cancer, the 8th edition (in Japanese). Tokyo: Kanehara-Shuppan, 2019.

7. The Papanicolaou society of cytopathology task force 
on standards of practice. Guidelines of the Papanicolaou society of cytopathology for the examination of fineneedle aspiration specimens from thyroid nodules. Diagn Cytopathol 1996;15:84-9.

8. The Japanese Society of Thyroid Surgery. General rules for the description of thyroid cancer, the 6th edition(in Japanese). Tokyo: Kanehara-Shuppan, 2005.

9. The Japan Thyroid Association. Guidelines for clinical practice for the management of thyroid nodules in Japan 2013 (in Japanese). Tokyo: Nankodo, 2013.

10. The Japanese Society of Thyroid Surgery. General rules for the description of thyroid cancer, the 7th edition (in Japanese). Tokyo: Kanehara-Shuppan, 2015.

11. Kumarasinghe P. Australian system for reporting thyroid cytology. In Kakdo K (editor). Thyroid cytology, differential diagnoses and pitfalls. Second edition. Singapore: Springer, 2019:69-76.

12. Kanematsu R, Hirokawa M, Higuchi M, et al. Risk of malignancy and clinical outcomes of cyst fluid only nodules in the thyroid based on ultrasound and aspiration cytology. Diagn Cytopathol 2020;48:30-4.

13. Takada N, Hirokawa M, Suzuki A, et al. Reappraisal of "cyst fluid only" on thyroid fine-needle aspiration cytology. Endocr J 2017;64:759-65.

14. Suzuki A, Hirokawa M, Higuchi M, et al. Evaluation of "benign" in thyroid Bethesda system (in Japanese). J Jpn Soc Clin Cytol 2014;53:257-63.

15. Cibas ES, Ali SZ. The Bethesda system for reporting thyroid cytopathology, Second edition. Switzerland: Springer, 2018.

16. Faquin WC, Wong LQ, Afrogheh AH, et al. Impact of reclassifying noninvasive follicular variant of papillary thyroid carcinoma on the risk of malignancy in The Bethesda System for Reporting Thyroid Cytopathology. Cancer Cytopathol 2016;124:181-7.

17. Pusztaszeri $M$, Bongiovanni $M$. The impact of noninvasive follicular thyroid neoplasm with papillary-like nuclear features (NIFTP) on the diagnosis of thyroid nodules. Gland Surg 2019;8:S86-97.

18. Maletta F, Massa F, Torregrossa L et al. Cytological features of "noninvasive follicular thyroid neoplasm with papillary-like nuclear features" and their correlation with tumor histology. Hum Pathol 2016;54:134-42.

19. Bongiovanni M, Faquin WC, Giovanella L, et al. Impact of non-invasive follicular thyroid neoplasms with papillarylike nuclear features (NIFTP) on risk of malignancy in patients undergoing lobectomy/thyroidectomy for suspected malignancy or malignant fine-needle aspiration cytology findings: a systematic review and meta-analysis. Eur J Endocrinol 2019;181:389-96.

20. Hirokawa M, Carney JA, Goellner JR, et al. Observer variation of encapsulated follicular lesions of the thyroid gland. Am J Surg Pathol 2002;26:1508-14.

21. Hirokawa M. Higuchi M. Suzuki A. et al. Prevalence and diagnostic significance of noninvasive follicular thyroid neoplasm with papillary-like nuclear features among tumors previously diagnosed as follicular adenoma: a single-institutional study in Japan. Endocr J 2020. [Epub ahead of print].

22. Hirokawa M, Higuchi M, Suzuki A, et al. Noninvasive follicular thyroid neoplasm with papillary-like nuclear features: a single-institutional experience in Japan. Endocr J 2017;64:1149-55.

23. Higuchi M, Hirokawa M, Kanematsu R. et al. Impact of the modification of the diagnostic criteria in the 2017 Bethesda system for reporting thyroid cytopathology: a report of a single institution in Japan. Endocr $\mathbf{J}$ 2018;65:1193-8.

24. Suzuki A, Hirokawa M, Nobuoka Y, et al. Evaluation of "unsatisfactory" in thyroid Bethesda system (in Japanese). J Jpn Soc Clin Cytol 2013;52:304-9.

25. Yamao N, Hirokawa M, Suzuki A. et al. Analysis of atypia of undetermined significance (AUS)/follicular lesion of undetermined significance (FLUS) in the Bethesda system for reporting thyroid cytopathology (BSRTC) (in Japanese). J Jpn Soc Clin Cytol 2014;53:342-8.

26. Higuchi $M$, Hirokawa $M$, Nobuoka Y. et al. An analysis of the Bethesda system for reporting thyroid cytopathology and a proposal for recommended clinical management (in Japanese). J Jpn Soc Clin Cytol 2012;51:395-401.

27. Kakudo K, Higuchi M, Hirokawa M. et al. Thyroid FNA Cytology in Asian practice-Active Surveillance for Indeterminate Thyroid Nodules Reduces Overtreatment of Thyroid Carcinomas. Cytopathology 2017;28:455-66.

28. Higuchi M, Hirokawa M, Yanase Y. et al. Cytology of follicular thyroid tumors, quality assurance, and differential diagnosis (in Japanese). J Jpn Soc Clin Cytol 2010;49:48-54.

29. Kihara M, Hirokawa M, Ito Y, et al. Final pathology findings after immediate or delayed surgery in patients with cytologically benign or follicular thyroid nodules. World J Surg 2011;35:558-62.

30. Higuchi M, Hirokawa M, Sasaki E, et al. Proposed algorithm for cytological diagnosis of thyroid follicular lesions and the reporting system. J Jpn Soc Clin Cytol 2014;53:264-70. 
31. Satoh S, Yamashita H, Kakudo K. Thyroid Cytology: The Japanese system and experience at Yamashita Thyroid Hospital. J Pathol Transl Med 2017;51:548-54.

32. Kakudo K, Kameyama K, Hirokawa M, et al. Subclassification of follicular neoplasms recommended by the Japan Thyroid Association reporting system of thyroid cytology. Int J Endocrinol 2015;2015:938305.

33. Vuong HG, Ngo HTT, Bychkov A. Differences in

Cite this article as: Hirokawa M, Suzuki A, Higuchi M, Hayashi T, Kuma S, Ito Y, Miyauchi A. The Japanese reporting system for thyroid aspiration cytology 2019 (JRSTAC2019). Gland Surg 2020;9(5):1653-1662. doi: 10.21037/gs-2019-catp-22 surgical resection rate and risk of malignancy in thyroid cytopathology practice between Western and Asian countries: a systematic review and meta-analysis. Cancer Cytopathol 2020;128:238-49.

34. Miyauchi A, Ito Y. Conservative Surveillance Management of Low-Risk Papillary Thyroid Microcarcinoma. Endocrinol Metab Clin North Am 2019;48:215-26. 